\title{
ULTRAVIOLET SPECTROSCOPY OF TYPE IIB SUPERNOVAE: DIVERSITY AND THE IMPACT OF CIRCUMSTELLAR MATERIAL
}

\author{
Sagi Ben-Ami ${ }^{1,2}$, Stephan Hachinger ${ }^{3,4}$, Avishay Gal-Yam ${ }^{2,13}$, Paolo A. Mazzali ${ }^{3,5,6}$, Alexei V. Filippenko ${ }^{7}$, \\ Assaf Horesh $^{2}$, Thomas Matheson ${ }^{8}$, Maryam Modjaz $^{9}$, Daniel N. Sauer ${ }^{10}$, Jeffrey M. Silverman ${ }^{11}$, Nathan Smith ${ }^{12}$, and \\ OFER YARON ${ }^{2}$ \\ ${ }^{1}$ Smithsonian Astrophysical Observatory, Harvard-Smithsonian Center for Astrophysics, 60 Garden St., Cambridge, MA 02138, USA; sbenami@cfa.harvard.edu \\ ${ }^{2}$ Department of Particle Physics and Astrophysics, The Weizmann Institute of Science, Rehovot 76100, Israel \\ ${ }^{3}$ Max-Planck-Institut für Astrophysik, Karl-Schwarzschild-Str. 1, D-85748 Garching, Germany \\ ${ }^{4}$ Institut für Theoretische Physik und Astrophysik, Universität Wurzburg, Emil-Fischer-Str. 31, D-97074 Wurzburg, Germany \\ 5 Astrophysics Research Institute, Liverpool John Moores University, Liverpool L3 5RF, UK \\ ${ }^{6}$ INAF-Osservatorio Astronomico, vicolo dell'Osservatorio, 5, I-35122 Padova, Italy \\ ${ }^{7}$ Department of Astronomy, University of California, Berkeley, CA 94720-3411, USA \\ ${ }^{8}$ National Optical Astronomy Observatory, 950 N. Cherry Avenue, Tucson, AZ 85719, USA \\ ${ }^{9}$ Center for Cosmology and Particle Physics, Department of Physics, New York University, 4 Washington Place, Room 529, New York, NY 10003, USA \\ ${ }^{10}$ Department of Astronomy, Stockholm University, Albanova University Center, SE-106 91 Stockholm, Sweden \\ ${ }^{11}$ Department of Astronomy, University of Texas, Austin, TX 78712, USA \\ ${ }^{12}$ Steward Observatory, University of Arizona, Tucson, AZ 85721, USA \\ Received 2014 November 6; accepted 2015 February 10; published 2015 April 13
}

\begin{abstract}
We present new Hubble Space Telescope (HST) multi-epoch ultraviolet (UV) spectra of the bright Type IIb SN 2013df, and undertake a comprehensive analysis of the set of four SNe IIb for which HST UV spectra are available (SN 1993J, SN 2001ig, SN 2011dh, and SN 2013df). We find strong diversity in both continuum levels and line features among these objects. We use radiative-transfer models that fit the optical part of the spectrum well, and find that in three of these four events we see a UV continuum flux excess, apparently unaffected by line absorption. We hypothesize that this emission originates above the photosphere, and is related to interaction with circumstellar material (CSM) located in close proximity to the SN progenitor. In contrast, the spectra of SN $2001 \mathrm{ig}$ are well fit by single-temperature models, display weak continuum and strong reverse-fluorescence features, and are similar to spectra of radioactive ${ }^{56} \mathrm{Ni}$-dominated SNe Ia. A comparison of the early shock-cooling components in the observed light curves with the UV continuum levels which we assume trace the strength of CSM interaction suggests that events with slower cooling have stronger CSM emission. The radio emission from events having a prominent UV excess is perhaps consistent with slower blast-wave velocities, as expected if the explosion shock was slowed down by the CSM that is also responsible for the strong UV, but this connection is currently speculative as it is based on only a few events.
\end{abstract}

Key words: supernovae: general - supernovae: individual (Type IIb)

\section{INTRODUCTION}

A core-collapse supernova (CC SN ) occurs when a massive star $\left(\gtrsim 8 M_{\odot}\right)$ ends its life in a terminal explosion that destroys the star completely and leaves a neutron star or a stellar-mass black hole as a compact remnant (e.g., Woosley \& Janka 2005). Classification of such supernovae $(\mathrm{SNe})$ is based primarily on their observed optical spectra, but also on their observed light curves (LCs; e.g., SNe IIP versus SNe IIL). SNe II have obvious hydrogen Balmer lines throughout their evolution, whereas SNe Ib lack prominent signatures of $\mathrm{H}$ while having strong He lines, and SNe Ic lack clear signatures of both $\mathrm{H}$ and He (e.g., Filippenko 1997). SNe IIb populate a unique location between the H-rich Type II and the stripped-envelope $\mathrm{SNe} \mathrm{Ib} / \mathrm{Ic}$ (Filippenko 1988, 1997).

Early-time spectra of Type IIb events are similar to those of SNe II, with strong Balmer lines dominating the optical spectrum. Later (i.e., days to weeks; Filippenko et al. 1993; Crockett et al. 2008; Bufano et al. 2014), the spectra evolve to resemble those of $\mathrm{SNe} \mathrm{Ib}$, with prominent $\mathrm{He}$ I lines. This behavior is attributed to the presence of a low-mass H-rich envelope-e.g., $0.18 M_{\odot}$ for SN 1993J, the best-studied SN IIb

\footnotetext{
${ }^{13}$ Kimmel Investigator.
}

to date (Woosley et al. 1994; Hachinger et al. 2012). Two common mechanisms that are invoked to explain the partial stripping of SN IIb progenitors are stellar winds from a massive star (e.g., SN 2001ig; Ryder et al. 2004) and mass loss through Roche-lobe overflow in a binary system (e.g., SN 1993J; Maund et al. 2004). The LCs of SNe IIb resemble those of radioactively powered $\mathrm{SNe} \mathrm{Ib}$ (Arcavi et al. 2012). In some cases, two peaks are observed in both the optical and the ultraviolet (UV; e.g., Filippenko et al. 1993; Arcavi et al. 2011; Van Dyk et al. 2014). The first peak is attributed to the shockcooling phase, and the second to radioactive nickel decay (e.g., Nakar \& Piro 2014).

For some nearby $\mathrm{SNe} \mathrm{IIb}$, analysis of Hubble Space Telescope (HST) images taken prior to the SN explosion led to the identification of the progenitor stars. In a few cases, namely SN 1993J, SN 2011dh, and SN 2013df, the progenitors were yellow supergiants (YSGs; $R=150-650 R_{\odot} ;$ Maund et al. 2004, 2011; Van Dyk et al. 2011, Van Dyk et al. 2014), somewhat hotter $(T \approx 6000 \mathrm{~K})$ than red supergiants, the progenitors of the more common SNe IIP (Smartt 2009). For SN 1993J, Maund et al. (2004) and Fox et al. (2014) find evidence for the presence of a hot (B2 Ia) star-a possible companion of the progenitor. Georgy (2012) shows, using stellar models including rotation, that $12-15 M_{\odot}$ main- 
sequence stars can end their lives as YSGs following increased mass-loss rates during their red supergiant phase. Smith et al. (2011) find that the statistics of SN subtypes require that binary interaction is a primary channel for stripped-envelope SNe.

Stancliffe \& Eldridge (2009) model the proposed binary system of the SN 1993J progenitor, in an attempt to understand whether stripping by a companion in a binary system might result in one of the stars becoming a YSG. In the case of SN 2011dh, Bersten et al. (2012) claim that evolution in a binary system has most likely caused the stripping as well. Using the "flash spectroscopy" method, Gal-Yam et al. (2014) demonstrate that the progenitor of the SN IIb 2013cu (iPTF13ast) likely had a surface composition similar to that of a late-type WN Wolf-Rayet (W-R) star (Smith \& Conti 2008).

Outside of the optical waveband, studies in the radio and Xray bands have probed the mass-loss history of these events in an attempt to uncover the progenitor stripping mechanism (e.g., Van Dyk et al. 1994; Fransson et al. 1996; Ryder et al. 2004; Chevalier \& Fransson 2006). Indications of circumstellar material (CSM) with a density flatter than $\rho_{w} \propto r^{-2}$ have been provided by radio-LC modeling, and X-ray emission from shocked CSM was found to be responsible for most of the Xray flux at early times (Fransson et al. 1996). Ryder et al. (2004, but see Soderberg et al. 2006) postulate that the observed modulations in the radio LC of SN2001ig are produced by density modulations in the CSM caused by motion in an eccentric binary system.

Chevalier \& Soderberg (2010) suggest that SNe IIb can be further divided into two subgroups. The first includes explosions of compact objects (Type cIIb, $R \approx 1 R_{\odot}$ ). These events are distinguished by weak optical emission from the shock-heated envelope at early times (i.e., adiabatic cooling phase), rapid expansion of the radio shell because of low CSM density resulting from high wind velocities, and nonthermal Xray emission from inverse-Compton scattering (Fransson et al. 1996). SN 2001ig and SN 2008ax are postulated to be members of this subgroup. In the case of SN 2008ax, the classification is also supported by the identification of a possible compact $\mathrm{W}-\mathrm{R}$ progenitor in pre-explosion $H S T$ images (Crockett et al. 2008), even though this conclusion is not as strong as in the cases of SN 1993J, SN 2011dh, and SN 2013df (a higher confidence level in the detection of the progenitor in pre-explosion images taken with HST). The second subgroup originates from explosions of cool extended progenitors surrounded by dense winds (Type eIIb, $R \gtrsim 100$ $\left.R_{\odot}\right)$. SN 1993J is the best-studied member of this group.

Despite the large number of investigations focused on $\mathrm{SNe} \mathrm{IIb}$, not much attention has been paid to the UV emission of these events, despite the flux excess some of them have exhibited compared to predicted theoretical models (e.g., Baron et al. 1993; Jeffery et al. 1994). This is partially because of the small number of events for which we have high-quality datasets in the UV band. In the following work we present early-time UV spectroscopy taken with HST for a sample of SNe IIb, with a focus on the recent SN 2013df, finding that UV spectra further illuminate the diversity observed in SNe IIb. We argue that the UV spectral energy distribution (SED) is governed by two parameters: the amount of CSM in the object's vicinity at the time of explosion, and the amount of ${ }^{56} \mathrm{Ni}$ synthesized and ejected in the explosion. We suggest that future division of $\mathrm{SNe}$ IIb will benefit by taking into account the observed UV
SED as an indicator of the presence of CSM around the progenitor, introducing another level of complexity to the picture suggested by Chevalier \& Soderberg (2010).

Section 2 describes the SN sample and observations used in this work, and the bolometric LCs are assembled. In Section 3 we analyze the bolometric LCs, identify dominant features in the observed spectra, construct radiative-transfer models, produce synthetic spectra, and compare these to HST observations. We present our conclusions and discuss their implications in Section 4.

\section{OBSERVATIONS}

\subsection{The Sample}

We analyze four SNe IIb having high signal-to-noise ratio UV spectra obtained with $H S T$ within 30 days after the estimated explosion. This is an exhaustive list; to the best of our knowledge, no other early-time UV spectra of SNe IIb have been obtained with $H S T$.

SN 2013df was discovered on 2013 June 7.78 (Ciabattari et al. 2013; UTC dates are used throughout this paper) in an outer spiral arm of NGC 4414 (distance $d=16.6 \pm 0.4 \mathrm{Mpc}$; Freedman et al. 2001). A comparison of $V$-band LCs of SN 1993J, SN 2011dh, and SN 2013df led Van Dyk et al. (2014) to estimate an explosion date of 2013 June 4, which we adopt throughput this paper (see also Morales-Garoffolo et al. 2014). Van Dyk et al. (2014) identify the SN progenitor in pre-explosion $H S T$ images, arguing for an extended star with an effective radius of $545 \pm 65 R_{\odot}$ and an effective temperature of $4250 \pm 100 \mathrm{~K}$. The progenitor is likely a YSG with an initial mass of $13-17 M_{\odot}$.

SN 1993J was discovered by amateur astronomer F. Garcia on 1993 March 28.9, at $d=3.6 \mathrm{Mpc}$ in the spiral galaxy M81 (Freedman et al. 1994). We adopt an explosion date of 1993 March 27.5 (Filippenko et al. 1993). The SN 1993J progenitor was identified in ground-based pre-explosion images by Aldering et al. (1994). The progenitor is a YSG with a radius of $\sim 600 R_{\odot}$ and a mass of 12-22 $M_{\odot}$ (Aldering et al. 1994; Maund et al. 2004; Fox et al. 2014). Maund et al. (2004) use photometric and spectroscopic observations taken $10 \mathrm{yr}$ after the explosion to give evidence for a massive star at the SN location, claimed to be the binary companion of the progenitor.

SN 2001ig was discovered on 2001 December 10.43 by Evans et al. (2001) in the spiral galaxy NGC 7424 at $d=11.5 \mathrm{Mpc}$ (Soria et al. 2006). We adopt an explosion date of 2001 December 3 based on radio LC modeling (Ryder et al. 2004). Using the same modeling, (Ryder et al. 2004) argue that the most likely progenitor of SN 2001ig is a W-R star with a radius of $\sim 10^{11} \mathrm{~cm}$. SN 2001ig is the only SN cIIb candidate for which early-time UV spectra were obtained with $H S T$.

SN 2011dh was discovered by amateur astronomer A. Riou on 2011 May 31.83 (Arcavi et al. 2011) in the Whirlpool galaxy (M51) at $d=8.05 \pm 0.35 \mathrm{Mpc}$ (Marion et al. 2014). SN 2011dh was not detected down to $g=21.44 \mathrm{mag}$ in Palomar Transient Factory images taken on 31.27 May 2011, and we adopt an explosion date of 31.5 May 2011 (Arcavi et al. 2011; Marion et al. 2014). Bersten et al. (2012) and Horesh et al. (2013) argue for an extended progenitor with a radius of $\sim 200 R_{\odot}$. This was verified by Van Dyk et al. (2013), who determined that the YSG at the SN location vanished in 
Table 1

Correlations Between UV SED and Other Observables in SNe IIb

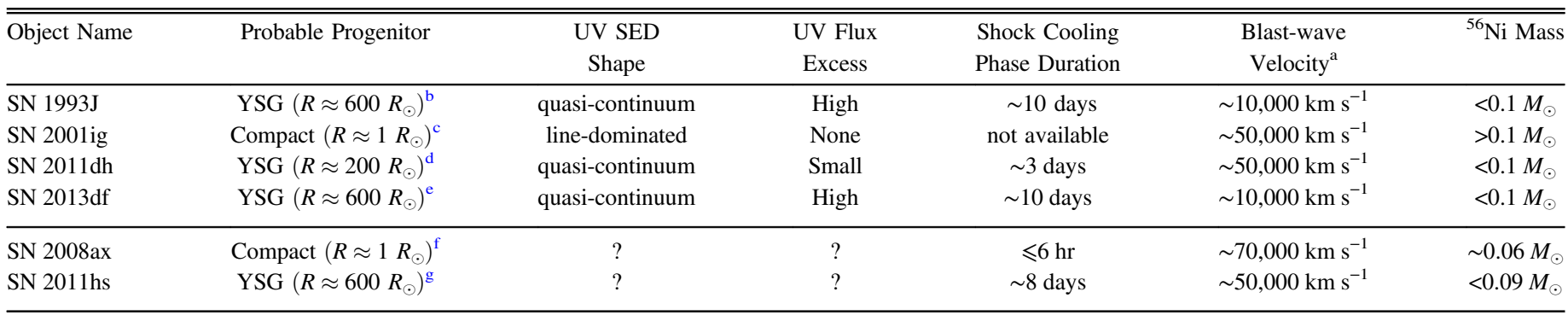

Note. See text for references. No early-time photometric data exist for SN 2001ig. However, SN 2008ax, which is also an SN cIIb candidate, shows no shock-cooling phase as early as $6 \mathrm{hr}$ after the estimated explosion (Crockett et al. 2008).

a e.g., Crockett et al. (2008), Horesh et al. (2013), Bufano et al. (2014).

b Aldering et al. (1994), Van Dyk et al. (2002).

${ }^{c}$ Ryder et al. (2004, 2006).

${ }^{d}$ Bersten et al. (2012), Horesh et al. (2013), Van Dyk et al. (2013).

e Van Dyk et al. (2014).

${ }^{\mathrm{f}}$ Crockett et al. (2008), Crockett et al. (2008).

${ }^{\mathrm{g}}$ Bufano et al. (2014).

post-explosion HST images; see also Maund et al. (2011) and Ergon et al. (2014a, 2014b).

We compare our LCs with those of two additional objects, SN 2008ax (Pastorello et al. 2008; Crockett et al. 2008) and SN 2011hs (Bufano et al. 2014), albeit these events have no early-time UV spectroscopy.

SN 2008ax was discovered independently by Mostardi, Li, and Filippenko on 2008 March 3.95, and by Nakano and Itagaki on 2008 March 5.12 (Nakano 2008), in the barred spiral galaxy NGC 4490 at $d=11.1 \mathrm{Mpc}$. Extremely tight limits on the explosion time of this event are placed by Arbour (2008), who monitored the host galaxy only $6 \mathrm{hr}$ before detection. We adopt an explosion date of 2008 March 3.7 (Pastorello et al. 2008, and references therein). SN 2008ax is a candidate Type cIlb event (Chevalier \& Soderberg 2010).

SN 2011hs was discovered on 2011 November 12.5 in the galaxy IC 5267 at $d=26.4 \mathrm{Mpc}$. We adopt an explosion date of 2011 November 6.5 based on a comparison of early-time photometric data with the SN 1993J LC (Bufano et al. 2014, and reference therein). Bufano et al. (2014) argue for an extended progenitor similar to the one observed for SN 1993J; see Section 3.1.

Table 1 lists all of these objects.

\subsection{HST Spectra}

We observed SN 2013df with HST on four epochs: 2013 June 17.2, 22.1, 26.1, and 30.2 (respectively $\sim 13,18,22$, and 26 days after the estimated explosion date; Program GO-13030, PI A. V. Filippenko). At each visit, three spectra of the SN were obtained: a UV spectrum using STIS/MAMA centered at $2376 \AA$, and two spectra using STIS/CCD centered at 4300 and $7751 \AA$. Data were reduced using the calstis software (Bostroem \& Proffitt 2011).

A UV+optical spectrum of SN 1993J was obtained on 1993 April 15.4 using $H S T$-FOS ( 19 days after the estimated explosion date; Program GO-4528, PI R. P. Kirshner; Jeffery et al. 1994). Data were reduced using the calfos software (Keyes et al. 1995).

UV+optical spectra of SN2001ig were taken on two different epochs, 2001 December 14 and 22.6, using HSTSTIS ( $\sim 11$ and $\sim 19$ days after the estimated explosion date;
Program GO-9114, PI R. P. Kirshner; Marion et al. 2014). Data were reduced using the calstis software (Bostroem \& Proffitt 2011).

A UV+optical spectrum of SN 2011dh was obtained with $H S T$-STIS/CCD on 2011 June 24.1 ( 24 days after the estimated explosion date; Program GO-12540, PI R. P. Kirshner; Marion et al. 2014). Data were reduced using the calstis software (Bostroem \& Proffitt 2011).

Table 2 summarizes all $H S T$ spectroscopic observations used in our analysis. Figure 1 shows the spectra described above, with insets highlighting the UV. Digital versions of these spectra are available from WISeREP (Yaron \& GalYam 2012).

\subsection{Bolometric LCs}

In order to set the context for the analysis of the UV emission, we first inspect the bolometric LCs of SNe IIb. We collected the LC of SN 1993J from Richmond et al. (1996), the LC of SN 2008ax from Pastorello et al. (2008), and the LC of SN 2011hs from Bufano et al. (2014).

In the case of SN 2011dh, the bolometric LC is taken from Marion et al. (2014). This LC starts four days after estimated explosion and does not include the initial adiabatic cooling phase. However, Arcavi et al. (2011) show that such a phase was detected using mostly unfiltered photometry from amateur astronomers (see Figure 2 inset). The duration of the shockcooling phase for SN 2011dh is less than 3 days (Arcavi et al. 2011); see also Ergon et al. (2014a, 2014b).

We construct a bolometric LC for SN 2013df from observations ${ }^{14}$ obtained with Swift-UVOT (UVW2, UVM2, and UVW1 bands), KAIT ( $B$ and $V$ bands), and RATIR $(r, i, z$, $J$, and $H$ bands). Our LC samples the period between 2013 June 13 and 2013 July 25 . Magnitudes were corrected for hostgalaxy and Milky Way extinction assuming $A_{V}=0.30 \mathrm{mag}$ and using the Cardelli et al. (1989) reddening law (see Van Dyk et al. 2014 for a thorough discussion of extinction estimates for SN 2013df). Magnitudes were converted to quasi-monochromatic flux at the effective wavelength of each filter. A quasisynthetic LC at each band was generated by fitting a spline to

\footnotetext{
${ }^{14}$ For LCs at specific bands, see Van Dyk et al. (2014).
} 
Table 2

HST Observations

\begin{tabular}{|c|c|c|c|c|c|}
\hline Object Name & $\begin{array}{l}\text { Date } \\
\text { (UT) }\end{array}$ & $\begin{array}{c}\text { Exposure Time } \\
\text { (s) }\end{array}$ & Instrument & Detector & Aperture/Grating \\
\hline \multirow[t]{9}{*}{ SN 2013df } & 2013 Jun 17.2 & 8476 & STIS & NUV-MAMA & $52 \times 0.2 / \mathrm{G} 230 \mathrm{~L}$ \\
\hline & 2013 Jun 17.4 & 222 & STIS & CCD & $52 \times 0.2 / \mathrm{G} 430 \mathrm{~L}$ \\
\hline & 2013 Jun 22.1 & 8594 & STIS & NUV-MAMA & $52 \times 0.2 / \mathrm{G} 230 \mathrm{~L}$ \\
\hline & 2013 Jun 22.3 & 108 & STIS & $\mathrm{CCD}$ & $52 \times 0.2 / \mathrm{G} 430 \mathrm{~L}$ \\
\hline & 2013 Jun 22.3 & 108 & STIS & $\mathrm{CCD}$ & $52 \times 0.2 / \mathrm{G} 750 \mathrm{~L}$ \\
\hline & 2013 Jun 26.3 & 108 & STIS & CCD & $52 \times 0.2 / \mathrm{G} 750 \mathrm{~L}$ \\
\hline & 2013 Jun 30.2 & 6118 & STIS & NUV-MAMA & $52 \times 0.2 / \mathrm{G} 230 \mathrm{~L}$ \\
\hline & 2013 Jun 30.3 & 118 & STIS & $\mathrm{CCD}$ & $52 \times 0.2 / \mathrm{G} 430 \mathrm{~L}$ \\
\hline & 2013 Jun 30.3 & 118 & STIS & $\mathrm{CCD}$ & $52 \times 0.2 / \mathrm{G} 750 \mathrm{~L}$ \\
\hline \multirow[t]{6}{*}{ SN 2001ig } & 2001 Dec 14.0 & 4343 & STIS & NUV-MAMA & $0.2 \times 0.2 / \mathrm{E} 140 \mathrm{M}$ \\
\hline & 2001 Dec 14.1 & 2703 & STIS & NUV-MAMA & $52 \times 0.2 / \mathrm{G} 230 \mathrm{~L}$ \\
\hline & 2001 Dec 14.2 & 200 & STIS & $\mathrm{CCD}$ & $52 \times 0.2 / \mathrm{G} 430 \mathrm{~L}$ \\
\hline & 2001 Dec 22.6 & 7907 & STIS & NUV-MAMA & $0.2 \times 0.2 / \mathrm{E} 140 \mathrm{M}$ \\
\hline & 2001 Dec 22.9 & 5514 & STIS & NUV-MAMA & $52 \times 0.2 / \mathrm{G} 230 \mathrm{~L}$ \\
\hline & 2001 Dec 22.6 & 300 & STIS & $\mathrm{CCD}$ & $52 \times 0.2 / \mathrm{G} 430 \mathrm{~L}$ \\
\hline \multirow[t]{3}{*}{ SN 1993J } & 15.4 Apr 1993 & 1800 & FOS & $\ldots$ & $4.3 / \mathrm{G} 160 \mathrm{~L}$ \\
\hline & 1993 Apr 15.5 & 1800 & FOS & $\ldots$ & $4.3 / \mathrm{G} 270 \mathrm{H}$ \\
\hline & 1993 Apr 15.5 & 1300 & FOS & $\ldots$ & $4.3 / \mathrm{G} 400 \mathrm{H}$ \\
\hline
\end{tabular}

Note. Digital data are available from the Weizmann Interactive Supernova Data Repository (WISeREP; Yaron \& Gal-Yam 2012; http://weizmann.ac.il/astrophysics/ wiserep/).

the measurements. In cases where the observations started on 2013 June 14 (KAIT $B$ and $V$ bands) and 2013 June 16 (RATIR $z H K$ ), an artificial data point was added on 2013 June 13 using backward extrapolation of the initial three measurements in each band. Varying this artificial point value by $20 \%$, we find that this method introduces an error of $<10 \%$ to the bolometric flux at this time. The monochromatic LCs were then summed using the trapezoidal integration method, with bins equal to the FWHM of each filter to derive a bolometric LC. In view of our well-sampled LC, and based on blackbody fits to the observed HST spectra, we estimate a coverage of $\geqslant 95 \%$ of the total bolometric flux, and no attempt was made to correct for emission below $2100 \AA$ or above $17,800 \AA$. The bolometric LCs are plotted in Figure 2.

\section{ANALYSIS}

\subsection{LC Comparison}

Comparing the bolometric LCs, we find that, with the exception of SN 2011hs, a similar width around peak magnitude is observed; see Figure 2. As noticed by Pastorello et al. (2008) for SN 2008ax and SN 1993J, this suggests a similar value for the $M_{\mathrm{ej}}^{3} / E_{k}$ ratio according to Arnett's law (Arnett 1982). The difference in luminosity is attributed to small changes in the amount of ${ }^{56} \mathrm{Ni}$ ejected in the explosion (e.g., Roming et al. 2009; Maurer et al. 2010; Perets et al. 2010; Ergon et al. 2014a).

The most significant difference between the observed LCs is the early contribution from the shock-heated envelope. Of the four events for which the shock-cooling phase duration can be estimated, SN 2011dh exhibits the fastest decay, followed by SN 2011hs, SN 1993J, and SN 2013df, in agreement with Morales-Garoffolo et al. (2014).

Nakar \& Piro (2014) argue that for progenitors with a loosely bound envelope and an envelope mass much smaller than the core mass, the cooling-phase duration is proportional to the amount of mass concentrated at the stellar radius. This suggests that the envelope of SN 2013df is more massive than that of, for example, SN 1993J. However, this does not agree with our findings from spectral modeling (see Figures 3 and 4), as well as with the conclusions of Morales-Garoffolo et al. (2014). We therefore suggest that perhaps an extended shockcooling phase might also be caused by the presence of CSM at the vicinity of the progenitor; see Sections 3.2 and 4. This scenario is also supported by the analysis of the $\mathrm{H} \alpha$ emission in nebular spectra presented by Morales-Garoffolo et al. (2014). In the case of SN 2008ax, the absence of a shock-cooling phase, despite early photometric measurements taken $<6 \mathrm{hr}$ from the time of explosion, led Pastorello et al. (2008) and Chevalier \& Soderberg (2010) to conclude that it had a compact progenitor $\left(R \approx 1 R_{\odot}\right)$. We note that this also likely 

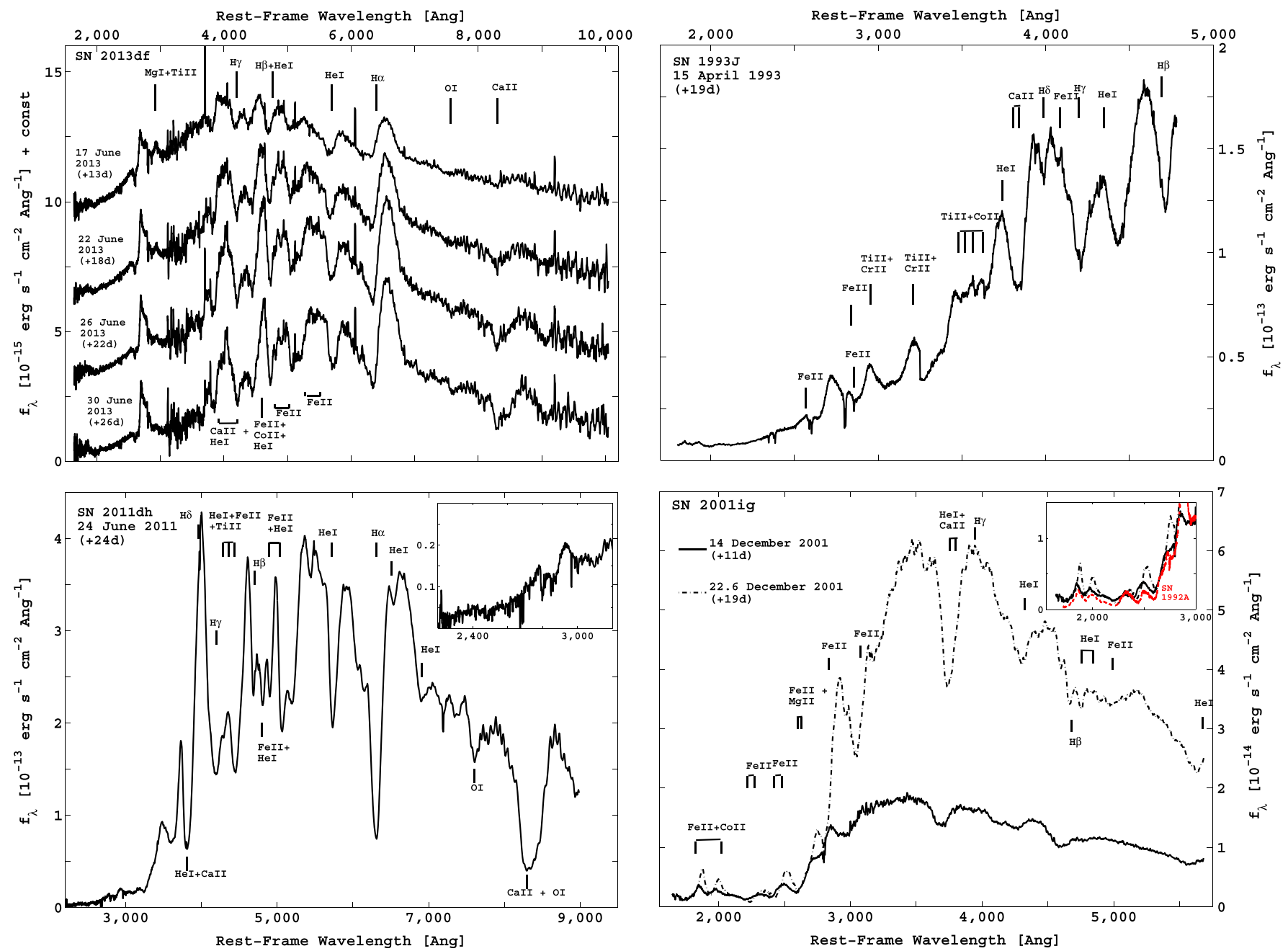

Figure 1. HST spectra of our SN IIb sample. Top left: SN 2013df (Program GO-13030, PI A. V. Filippenko). Top right: SN 1993J (Program GO-4528, PI R. P. Kirshner; Jeffery et al. 1994). Bottom left: SN 2011dh (Program GO-12540, PI R. P. Kirshner; Marion et al. 2014). Bottom right: SN 2001ig (Program GO-9114, PI R. P. Kirshner; Marion et al. 2014). The inset shows a comparison with the UV spectrum of the SN Ia 1992 A (Program GO-4016, PI R. P. Kirshner; Kirshner et al. 1993). Line identification is based on the spectral model described in Section 3.3. In the case of SN 2013df, we can model the strong, broad, asymmetric line at around $2800 \AA ̊$ using SYNOW (Mg II, Co II, Fe II, and Ti II at a temperature of 10,000 K; Branch et al. 2007; Parrent et al. 2010). However, this exercise introduces other lines into the modeled spectra below 2800 A which we do not observe. Digital data are available from the Weizmann Interactive Supernova Data Repository (WISeREP; Yaron \& Gal-Yam 2012; http://weizmann.ac.il/astrophysics/wiserep/).

means it did not have dense CSM, and may also argue against a nearby binary companion (Crockett et al. 2008).

Two events that sample the lower and upper energy regimes in our sample are SN2011hs and SN 2001ig (Ryder et al. 2004, 2006; Bufano et al. 2014). In the case of $\mathrm{SN} 2011 \mathrm{hs}$, the estimated ${ }^{56} \mathrm{Ni}$ mass and the energy are $0.04 M_{\odot}$ and $8 \times 10^{50} \mathrm{erg}$, respectively. An extended progenitor $\left(R \approx 550 R_{\odot}\right)$ is most likely based on the observed initial decline in the LC (Bufano et al. 2014). For SN 2001ig, no reliable photometric data were found. We therefore integrate over the $H S T$ spectra in the wavelength range 5000-5800 $\AA$ to create two data points in the $V$ band, and compare this result to the $V$-band LC of SN 2008ax and SN 2013df (Figure 2, inset). SN 2001ig is the most luminous event in our sample in $V$. Silverman et al. (2009) suggest that $0.13 M_{\odot}$ of ${ }^{56} \mathrm{Ni}$ was ejected by this event, slightly lower than predicted by the peak $V$ versus Ni-mass correlation of Perets et al. (2010), if we assume the second data point represents the SN peak magnitude. The effect of the higher luminosity on the UV emission from this event is discussed in Section 3.2.

\subsection{Observed Spectra}

SN 2013df spectra longward of $\sim 4000 \AA$ exhibit a continuum with strong absorption lines, as expected in the photospheric phase of CC SNe. The spectra are dominated ${ }^{15}$ by $\mathrm{H}$ Balmer lines $(6563,4861,4340 \AA)$, He $\mathrm{I}$ lines $(5875$, $4471,3888 \AA)$, Ca II H\&K $(3968,3934 \AA)$, the Ca II nearinfrared triplet $(8662,8542,8498 \AA)$, the O I triplet $(7774 \AA)$, and many features from iron-group elements (e.g., Fe II lines at 5316, 5276, $4583 \AA$ and Co II at $4660 \AA$ ). . Below $~ 4000 \AA$, all spectra show an excess of emission with respect to a singletemperature model. The SED at these wavelengths is smooth, with a strong, broad, asymmetric line at around $2800 \AA$. Using SYNOW (Branch et al. 2007; Parrent et al. 2010), we can model the observed line profile with $\mathrm{Mg}$ II, Co II, Fe II, and Ti II at a temperature of $10,000 \mathrm{~K}$. However, this exercise introduces other lines into the modeled spectra below $2800 \AA$ which we do not observe.

${ }^{15}$ Line identification is based on the spectral model described in Section 3.3 . 


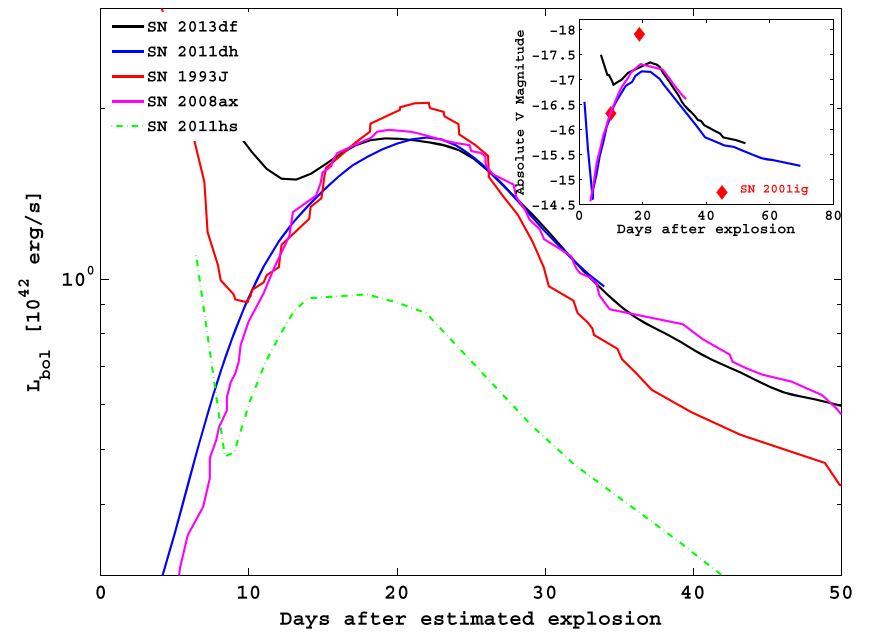

Figure 2. Bolometric LC comparison. Similar behavior is exhibited in all cases. The variations are caused by a different mass of ${ }^{56} \mathrm{Ni}$ ejected in each event, the shock-breakout radius, and the progenitor density profile (Perets et al. 2010; Nakar \& Piro 2014). Bolometric LCs are taken from Richmond et al. (1996; SN 1993J), Pastorello et al. (2008; SN 2008ax), Marion et al. (2014; SN 2011dh), and Bufano et al. (2014; SN 2011hs). Inset: a $V$-band comparison suggests that SN 2001ig is the intrinsically brightest event in our sample (no bolometric data exist). The inset also shows the initial shockcooling phase for SN 2011dh (based on unfiltered measurements by amateur astronomers; see Arcavi et al. 2011).

The SN 1993J spectrum taken on 1993 April 15 is dominated by $\mathrm{H}$ Balmer lines at $11,000 \pm 1000 \mathrm{~km} \mathrm{~s}^{-1}$, He I $\lambda \lambda 4471,4921$ at $9000 \pm 1000 \mathrm{~km} \mathrm{~s}^{-1}$, and the Ca II $\mathrm{H} \& \mathrm{~K}$ lines at $9000 \mathrm{~km} \mathrm{~s}^{-1}$. Iron-group element lines (e.g., Fe II, Ti II, and $\mathrm{Cr}$ II) are also present all across the observed spectrum. SN 1993J exhibits a smooth continuum below $\sim 3000 \AA$ similar to the one observed in SN 2013df, with some features of iron-group elements identified above the continuum; for further details, see Section 3.3 as well as Baron et al. (1993).

The SN 2011dh spectrum taken on 2011 June 24 is dominated by $\mathrm{Ca}$ II $\mathrm{H} \& \mathrm{~K}$ and the $\mathrm{Ca}$ II near-IR triplet at $9000 \pm 400 \mathrm{~km} \mathrm{~s}^{-1}, \quad \mathrm{H}$ Balmer lines at velocities of $10,000 \pm 250 \mathrm{~km} \mathrm{~s}^{-1}$, He I lines (3888, 4471, 4921, 5015, 5875,6678 , and $7065 \AA$ ) at velocities of $7600 \pm 200 \mathrm{~km} \mathrm{~s}^{-1}$, and the $\mathrm{O}_{\mathrm{I}}$ triplet $(7774 \AA)$ at a velocity of $6700 \mathrm{~km} \mathrm{~s}^{-1}$. Contributions from $\mathrm{Fe}, \mathrm{Ti}$, and other iron-group elements are also evident all across the observed spectrum at velocities of $7500 \pm 500 \mathrm{~km} \mathrm{~s}^{-1}$. At UV wavelengths, the SED is smooth and featureless as in SN 2013df and SN 1993J, but the flux excess is significantly lower than in the latter two cases (Figure 1, bottom-left panel).

SN 2001ig spectra taken on 14 and 2001 December 22 (11 and 19 days past the estimated explosion date) are dominated by He I lines $(3888,4471,4921,5012,5875 \AA)$, Fe II $\lambda 5169$, and $\mathrm{H}$ Balmer lines. The contribution from $\mathrm{Ca}$ II to the feature around $3900 \AA$ can also be inferred from the analysis done on the optical and near-IR spectra (Silverman et al. 2009). At shorter wavelengths, absorption by iron-group elements, mainly Fe II and Co II lines, dominates the SED. All of the observed features strengthen in the later spectrum. Heavyelement lines point to a high expansion velocity of the ejecta $\left(\sim 10,000 \mathrm{~km} \mathrm{~s}^{-1}\right)$, while the $\mathrm{H}$ and $\mathrm{He}$ lines are observed at velocities of $\sim 13,000 \mathrm{~km} \mathrm{~s}^{-1}$.

\subsection{Spectral Models}

In order to study the ejecta structure of SN 2013df (abundances, density profile), we calculate radiative-transfer models. In addition to modeling the four UV-optical spectra we have of SN 2013df, we also modeled the other SNe in our UV spectroscopic sample.

Modeling SN 2013df, we assume approximate spherical symmetry and follow the "abundance tomography" approach of Mazzali et al. (2014). We assume a density profile for the $\mathrm{SN}$ and then infer an abundance stratification from photospheric spectra in the phase of homologous, force-free expansion. Doing this, we exploit the fact that the photosphere recedes with time. Early-time spectra show the imprint of the outermost layers of the envelope, and later spectra are dominated by material further inward. Thus, starting from reasonable initial values, the abundances can be inferred step by step. The earliest spectrum is used to determine optimum-fit abundances in the outer layers, and later spectra to subsequently find the abundances in the layers below, while keeping the abundances in the outer layers fixed. This process requires iteration in order to be optimized. We have repeated the fitting procedure several times with different density profiles in order to ensure that the profile finally used reasonably describes the actual envelope.

The spectral synthesis is performed with our spherically symmetric Monte Carlo radiative-transfer code (Mazzali et al. 1993; Lucy 1999; Mazzali 2000a), including abundance stratification (Stehle et al. 2005) and a module that calculates the ionization of $\mathrm{H}$ and $\mathrm{He}$ in full non-local thermodynamic equilibrium (NLTE; Hachinger et al. 2012). The code calculates the radiation field within a spherically symmetric SN expanding "atmosphere," producing synthetic spectra. As input parameters, it reads the abundance and density stratification, as well as the luminosity of the $\mathrm{SN}$, and the radius/ velocity of the "photosphere" (the lower boundary of the atmosphere) at each epoch $t$. The code simulates the propagation of energy packets, starting from the photosphere where a blackbody spectrum is assumed to be emitted. The packets interact with atomic lines and free electrons. The state of the plasma, which is needed to calculate these interactions, is computed in NLTE for $\mathrm{H}$ and He. Our NLTE module (Hachinger et al. 2012) takes into account heating by Compton electrons produced by $\gamma$-rays from ${ }^{56} \mathrm{Ni}$ and ${ }^{56} \mathrm{Co}$ decay, using the methods of Lucy (1991). The required heating rates are computed using a modified version of the LC code developed by P. A. Mazzali and used by Cappellaro et al. (1997). In order to arrive at a consistent solution for both the radiation field and plasma, the radiation Monte Carlo routine and the plasma state routine of the code are iteratively called in turn. After convergence, an output spectrum is calculated from the formal integral of the transfer equation.

The density model we use for SN 2013df is derived from model 13C of Woosley et al. (1994), who set up a model sequence to explain the prototypical SN 1993J. Model 13C has been successfully used by Tsvetkov et al. (2009) to model SN 2008ax. It turns out that the high-velocity tail of $13 \mathrm{C}$ produces somewhat too strong $\mathrm{H}$ lines. Therefore, we tried different ad-hoc modifications of the model (mostly downscaling the density above a certain threshold velocity/radius). A model that describes SN 2013df well was obtained by scaling the density above $11,000 \mathrm{~km} \mathrm{~s}^{-1}$ to one half of its original 


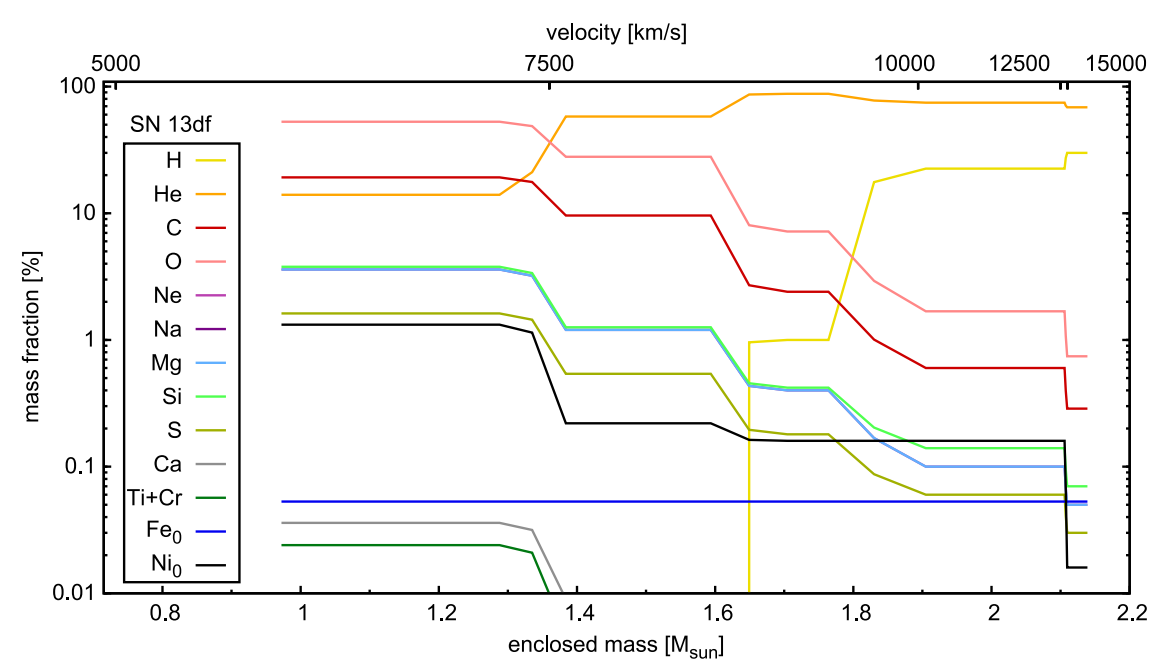

Figure 3. SN 2013df mass stratification for the 2013 June 30 model. Only the outermost $0.1-0.2 M_{\odot}$ of our envelope is H-enriched; the material below consists mostly of $\mathrm{He}$ and $\mathrm{C} / \mathrm{O}$ in the deeper layers. Traces of iron-group elements are also present at high velocities (i.e., above $10,000 \mathrm{~km} \mathrm{~s}^{-1}$ ).

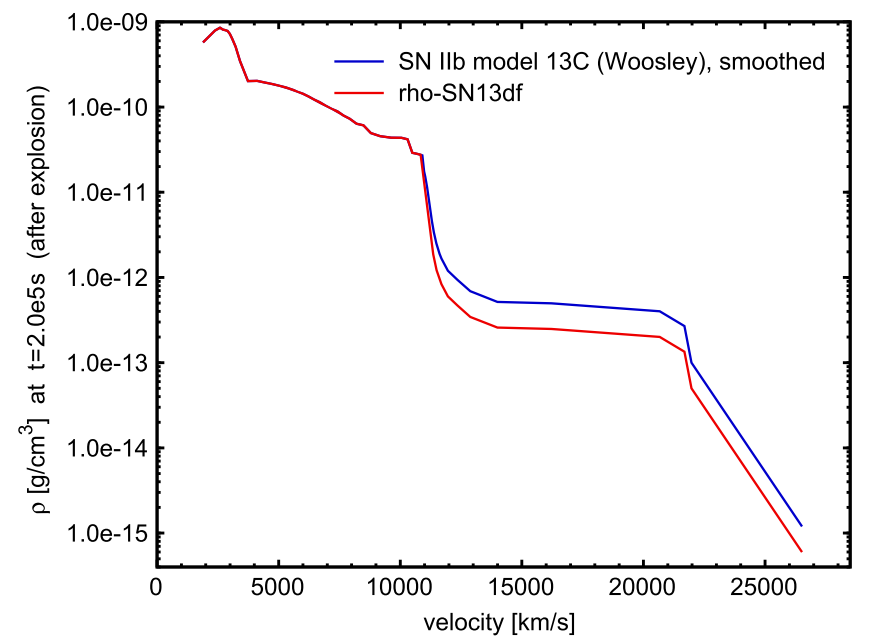

Figure 4. SN 2013df model density profile at $2 \times 10^{5} \mathrm{~s}(\sim 2.3$ days $)$ after explosion. A model that well describes SN 2013df was obtained by scaling the density above $11,000 \mathrm{~km} \mathrm{~s}^{-1}$ to one half of its original value (red curve).

value; see Figure 4. The original $13 \mathrm{C}$ model (Woosley et al. 1994) assumes a progenitor mass of $3.7 M_{\odot}\left(1.5 M_{\odot}\right.$ of which end up in the remaining neutron star), and our model is only slightly less massive (by $0.04 M_{\odot}$ ). The explosion energy in $13 \mathrm{C}$ was $1.2 \times 10^{51} \mathrm{erg}$, which is reduced to $1.1 \times 10^{51} \mathrm{erg}$ in our model. Our estimate of the progenitor mass at the time of explosion is higher than the one derived using the relation between the He-core mass and main sequence mass (Sugimoto \& Nomoto 1980) by Morales-Garoffolo et al. (2014). We argue that the overall characteristics of SN 2013df are more in agreement with SN 1993J than with SN $2011 \mathrm{dh}$ (e.g., SED and duration of shock-cooling phase), and therefore a progenitor mass similar to that of SN 1993J is more plausible. Moreover, our explosionenergy estimate is similar to that derived by Morales-Garoffolo et al. (2014), and assuming a lower progenitor mass at the time of explosion results in higher expansion velocities in our models than the expansion velocities observed.

The abundances we assume as a starting point are taken from adequate zones of the models for SN 2008ax presented by Hachinger et al. (2012). Only the outermost $0.1-0.2 M_{\odot}$ of our envelope are $\mathrm{H}$-enriched; the material below mostly consists of
$\mathrm{He}$ (and $\mathrm{C} / \mathrm{O}$ in the deeper layers). Taking into account the lower density profile we adopt (Figure 4), this suggests a lower H-envelope mass for SN 2013df in comparison to SN 1993J. Under these constraints, we optimized the abundances, inferring the best-fitting values. The synthetic spectra obtained using our radiative-transfer code are given in Figure 5. Our models suggest a blackbody temperature of $8200 \mathrm{~K}$ and an effective photosphere temperature of $5650 \mathrm{~K}$ in the spectrum taken on 2013 June 17, dropping to a blackbody temperature of $6800 \mathrm{~K}$ and an effective photosphere temperature of $5300 \mathrm{~K}$ in the spectrum taken on 2014 June 30, in agreement with Morales-Garoffolo et al. (2014). The photospheric velocity also drops from 9600 to $5900 \mathrm{~km} \mathrm{~s}^{-1}$. Velocity-space stratification for the model describing the spectrum taken on 2013 June 30 is given in Figure 3. Only the outermost $0.1-0.2 M_{\odot}$ of our envelope are $\mathrm{H}$-enriched; the material below mostly consists of $\mathrm{He}$ and $\mathrm{C} / \mathrm{O}$ in the deeper layers. Traces of iron-group elements are also present at high velocities (i.e., above $10,000 \mathrm{~km} \mathrm{~s}^{-1}$ ).

We find a good match between the observed and synthetic spectra at wavelengths above $\sim 5000 \AA$. At shorter wavelengths, our models simulate most of the observed lines (mainly $\mathrm{Fe}_{\text {II }}$ and $\mathrm{Ca}$ II), but the observed spectrum seems to include an additional component that is not affected by line absorption and is responsible to most of the emitted flux. This trend is consistent in all four simulated spectra, and is stronger at shorter wavelengths. In an attempt to increase the UV flux, which in our models is absorbed by iron-group elements in the $\mathrm{UV}(\mathrm{Fe}, \mathrm{Ti}, \mathrm{Cr})$, we have lowered the metal content in the outer layers of the ejecta, but were unable to reproduce the observed iron-group element lines above $\sim 4000 \AA$. Increasing the metal content in the outer layers to allow higher probability for reverse fluorescence induces line structure in the UV that is not present in the observed spectra. We therefore conclude that the source of the UV emission is above the photosphere, and is most likely associated with CSM shocked by the SN blast wave. This argument is supported by the flat UV LC after the first peak, as well as by the nebular spectral analysis, both presented by Morales-Garoffolo et al. (2014).

The models for SNe 1993J, 2001ig, and 2011dh are set up as minimal modifications of our SN 2013df model. We have chosen this strategy, partly justified by the observed similarities in the bolometric LCs (Section 3.1), so as to bring out the 

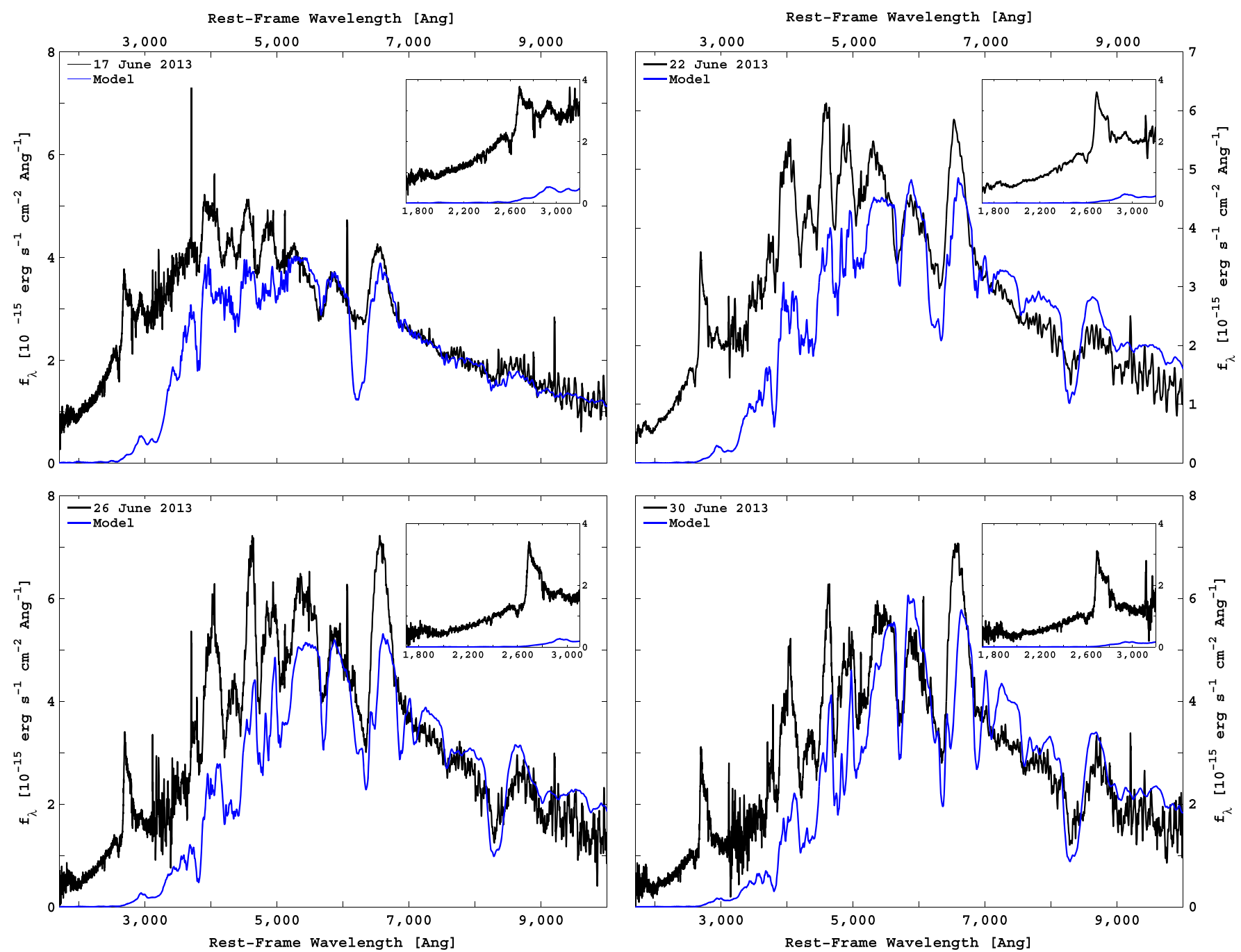

Figure 5. SN 2013df synthetic spectra. We get a good match above $5000 \AA$ A. At shorter wavelengths, an additional component that is not affected by line absorption from iron-group elements seems to be present. We associate this component with emission from shocked CSM above the photosphere.

principal physical changes from one to the other $\mathrm{SN}-$ even if more detailed differences in the density structure, etc, between the SNe may thus not be captured in our study.

With this approach, one spectrum of each comparison $\mathrm{SN}$ is sufficient in order to allow for a basic comparison. We started out rerunning the SN 2013df model with the modified values for epoch and basic SN parameters (such as distance and reddening), and a starting guess for photospheric velocity and luminosity. Usually, we arrived at a well-fitting model upon further optimizing the latter two parameters, and only slightly changing the abundance stratification.

For SN 1993J, a detailed analysis of the spectrum based on the Woosley et al. (1994) 13 C model we have used can be found in Woosley et al. (1994). The model suggests a blackbody temperature of $8200 \mathrm{~K}$, a photosphere effective temperature of $5900 \mathrm{~K}$, and a photosphere velocity of $8250 \mathrm{~km} \mathrm{~s}^{-1}$. For SN 2011dh, our models suggest a blackbody temperature of $7470 \mathrm{~K}$ and an effective photospheric temperature of $5900 \mathrm{~K}$, slightly lower than the results of Ergon et al. (2014a). The photospheric velocity we infer is $6750 \mathrm{~km} \mathrm{~s}^{-1}$, in agreement with Ergon et al. (2014a).

For both SN 1993J and SN 2011dh, we find a similar trend to the one observed for SN 2013df, in which an additional component not affected by line absorption is responsible for most of the emission below $\sim 4000 \AA$. The relative amount of flux attributed to this component is weakest in the case of SN 2011dh, and similar in the case of SN 1993J, as seen in the insets of Figure 6.

In the case of SN 2001ig, our models suggest a photosphere velocity of $8750 \mathrm{~km} \mathrm{~s}^{-1}$, a photospheric temperature of $7000 \mathrm{~K}$, and a blackbody temperature of $12,000 \mathrm{~K}$, in agreement with Silverman et al. (2009). The UV flux ratio below $3000 \AA$ is much lower. Iron-group element lines are clearly seen in this wavelength range, and we obtain a good match between our synthetic spectrum and the observed one at all wavelengths; see Figure 7. In addition, the line strength in the UV is directly proportional to the flux at longer wavelengths, as seen by comparing the spectra taken on 2001 December 14 (11 days after estimated explosion) and on 2001 December 22 (19 days after estimated explosion); see the bottom-right panel of Figure 1. We conclude that the SN 2001ig UV flux originates from reverse fluorescence of photons at longer wavelengths by excited iron-group elements in the expanding ejecta, and is similar to the observed UV spectrum of $\mathrm{SNe}$ Ia (e.g., Panagia 2007; Hachinger et al. 2012; Mazzali et al. 2014); see also Silverman et al. (2013) for a discussion of SNe Ia 

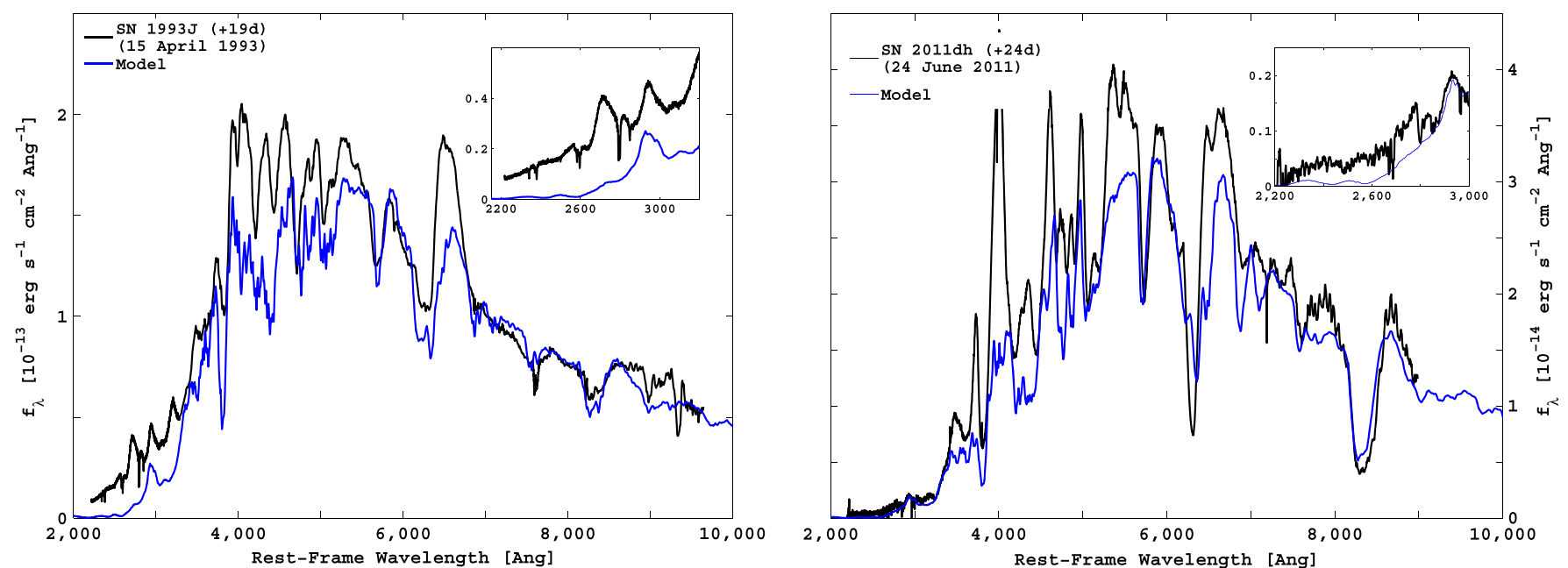

Figure 6. SN 1993J and SN 2011dh synthetic spectra. We obtain similar results to those for SN 2013df. The amount of flux excess compared to our synthetic models varies between events.

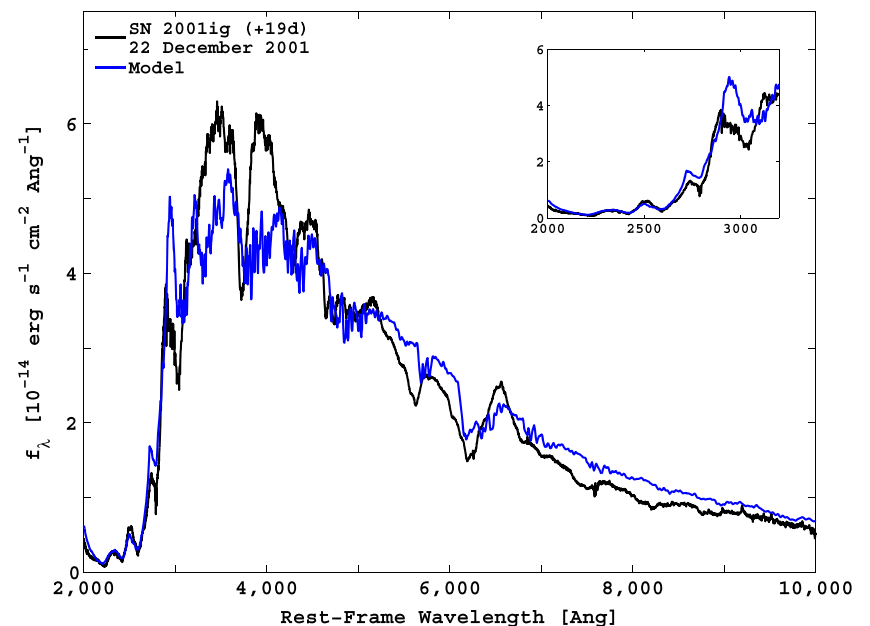

Figure 7. SN 2001ig synthetic spectrum. We achieve a good match at all wavelengths. The mechanism producing the observed UV flux is reverse fluorescence of photons at longer wavelengths by excited iron-group element atoms. The same mechanism is responsible for the observed UV emission in SNe Ia (e.g., Hachinger et al. 2012).

interacting with CSM. As mentioned in Section 3.1, this $\mathrm{SN}$ is intrinsically the brightest event in our sample, and it synthesized the most ${ }^{56} \mathrm{Ni}$ (Silverman et al. 2009).

\section{DISCUSSION}

Prior work has investigated diversity in the optical spectra and in emission at X-ray and radio bands among $\mathrm{SNe} \mathrm{IIb}$. Based on these observables, Chevalier \& Soderberg (2010) claim there is an internal division between $\mathrm{SNe}$ IIb having compact progenitors and those with extended progenitors. Studies of pre-explosion images from the HST archive show that indeed SN 1993J, SN 2011dh, and SN 2013df had YSG progenitors (e.g., Aldering et al. 1994; Maund et al. 2011; Van Dyk et al. 2013, 2014; Ergon et al. 2014a), while observations of the SN 2008ax progenitor are consistent with a compact $\mathrm{W}-\mathrm{R}$ star, though this is inconclusive (Crockett et al. 2008). Ryder et al. (2004) suggest that SN 2001ig might also belong to the class of $\mathrm{SNe}$ IIb with a compact progenitor, based on analysis of data in the radio waveband.
In this study we have examined early-time UV spectra of $\mathrm{SNe}$ IIb in an attempt to find whether the UV band holds information that might further reveal the nature of $\mathrm{SN} \mathrm{IIb}$ progenitors and their vicinity. We have gathered a sample of four events for which high-quality UV spectra were obtained within 30 days of the time of estimated explosion, and using radiative-transfer models we investigate the spectral diversity in the UV band between these events.

We find that our sample can be divided into two groups. The first group includes SN 1993J, SN 2011dh, and SN 2013df, all associated with extended YSG progenitors $\left(\sim 10^{13} \mathrm{~cm}\right)$, with expected low-velocity winds (e.g., Bufano et al. 2014, and reference therein). For these three events we find a UV continuum flux excess, not affected by line absorption. We conclude it most likely originates above the photosphere and is the result of shocked low-metallicity CSM. ${ }^{16}$ The flux excess varies between events. It is strongest in the case of SN 2013df and weakest in the case of SN 2011dh. Comparing the duration of the shock-cooling phase among the three $\mathrm{SNe}$, we find a correlation between the shock-cooling phase duration and the amount of flux in the UV attributed to the shocked CSM: the duration of the shock-cooling phase is longest in the case of SN 2013df, and shortest for SN 2011dh. This seems to suggest that the shock-cooling phase is affected not only by the relative amount of mass at the outer layers of the progenitor (Nakar \& Piro 2014), but also by the presence of dense CSM at the progenitor vicinity at the time of explosion. The presence of dense CSM will also affect the blast-wave velocity derived through observed peak spectral radio luminosities ${ }^{17}$ (Chevalier \& Soderberg 2010; Horesh et al. 2013; Bufano et al. 2014). A comparison reveals that SN 2011dh had a higher velocity than SN 1993J, while SN 2013df had a velocity similar ${ }^{18}$ to the one

\footnotetext{
${ }^{16}$ Note that prominent emission lines of $\mathrm{H}$ and $\mathrm{He}$ are not always expected from CSM interaction, in case the mass loss is not constant (Moriya \& Tominaga 2012).

17 A unique event in this context is SN 2003bg (Hamuy et al. 2009; Mazzali et al. 2009), which exhibits a high blast-wave velocity attributed to the presence of CSM, but with no indication for an initial shock-cooling phase. SN 2003 bg was classified as a Type IIb broad-line SN, and is more energetic than the events analyzed in this work-further expanding the diversity of SNe IIb. 18 Private correspondence with A. Soderberg and A. Kamble. Radio data of SN 2013df are not public at the time of the writing of this paper; see a future paper by A. Kamble et al. (in preparation).
} 
measured for SN 1993J. A comparison between SN 2011dh and SN 2011hs further illustrates the diversity in this class of events. We suggest that $\mathrm{SN} 2011 \mathrm{hs}$ is an intermediate case, with a CSM amount in between that of SN $2011 \mathrm{dh}$, and SN 2013df. This interpretation is supported by the coolingphase duration and the inferred blast-wave velocity (Table 1), but is speculative because the lack of early-time UV spectra of SN 2011hs does not allow us to reach a clear conclusion. Future observations of SNe IIb (specifically, early-time UV spectroscopy) will allow us to develop a more quantitative understanding of the effect of CSM on observables such as shock cooling phase duration and blast-wave velocity.

In the case of SN 2001ig, the observed UV spectra display a weak continuum and strong reverse-fluorescence features, similar to the UV spectra of $\mathrm{SNe}$ Ia such as $\mathrm{SN} 1992 \mathrm{~A}$ (Kirshner et al. 1993). A comparison of $V$-band LCs shows that indeed SN 2001ig was the most luminous event in our sample, and so the photon budget in the visible band was higher, leading to a higher probability for reverse fluorescence to occur. We argue that the observed similarity to SNe Ia reflects a high ratio of radioactive nickel mass to total mass and supports the inclusion of this event among the group of SNe IIb originating from a compact progenitor.

Our analysis suggests a correlation between the UV SED, blast-wave velocity, shock-cooling phase, and ejected ${ }^{56} \mathrm{Ni}$ mass; see Table 1. The first three observables seem to be affected by the amount of CSM around the progenitor at the time of explosion, while the amount of ${ }^{56} \mathrm{Ni}$ mass ejected affects the shape of the SED (i.e., quasi-continuum versus linedominated). If the CSM interaction UV emission modifies the early LC shape, it could lead to derivation of inaccurate progenitor radii using the methods of, e.g., Nakar \& Piro (2014) that do not account for such effects. This could explain the slight discrepancies in progenitor radius estimates observed by Van Dyk et al. (2014), as one method used is affected by the presence of CSM (i.e., progenitor radius based on shockcooling phase), while the other is not (i.e., progenitor radius based on HST images). In a similar manner, the presence of CSM can explain the large discrepancy between the progenitor radius estimates obtained by Morales-Garoffolo et al. (2014) using the methods of Nakar \& Piro (2014), and those of Van Dyk et al. (2014).

Our findings extend those of Chevalier \& Soderberg (2010), showing the diversity within the group of SNe IIb having an extended progenitor, and add emission in the UV band to the list of observables that might help us in understanding this diversity. Most notably, we show a correlation between the UV SED and observables related to the progenitor and its environment. Our findings are limited by our small sample size, in which only a single event is assumed to belong to the class of SNe IIb originating from a compact progenitor. Future work should therefore aim to increase the number of $\mathrm{SNe} \mathrm{IIb}$ for which we do have early-time high-quality UV data simultaneously with data across the electromagnetic spectrum in an attempt to better constrain the type of progenitors and the processes that lead to this important class of CC SNe.

S.B. acknowledges support for this work by NASA through Einstein Postdoctoral Fellowship grant number PF0000 awarded by the Chandra X-ray Center, which is operated by the Smithsonian Astrophysical Observatory for NASA under contract NAS8-03060. S.H. is supported by a Minerva
ARCHES award. A.G. acknowledges support by grants from the ISF, BSF, GIF, Minerva, FP7/ERC grant \#307260, the "Quantum-universe" I-core program of the planning and budgeting committee and the ISF, and a Kimmel Investigator award. A.V.F. and his group at UC Berkeley have depended on generous financial assistance from Gary \& Cynthia Bengier, the Richard \& Rhoda Goldman Fund, the Sylvia \& Jim Katzman Foundation, the Christopher R. Redlich Fund, the TABASGO Foundation, NSF grant AST-1211916, and NASA/ HST grant GO-13030 from the Space Telescope Science Institute (which is operated by the Association of Universities for Research in Astronomy, Inc., under NASA contract NAS 05-26555). J.M.S is supported by an NSF Astronomy and Astrophysics Postdoctoral Fellowship under award AST1302771.

\section{REFERENCES}

Aldering, G., Humphreys, R. M., \& Richmond, M. 1994, AJ, 107, 662 Arbour, R. 2008, CBET, 1286, 2

Arcavi, I., Gal-Yam, A., Cenko, S. B., et al. 2012, ApJL, 756, L30

Arcavi, I., Gal-Yam, A., Yaron, O., et al. 2011, ApJL, 742, L18

Arnett, W. D. 1982, ApJ, 253, 785

Baron, E., Hauschildt, P. H., Branch, D., et al. 1993, ApJL, 416, L21

Bersten, M. C., Benvenuto, O. G., Nomoto, K., et al. 2012, ApJ, 757, 31

Bostroem, K., \& Proffitt, C. 2011, in STIS Data Handbook, HST Data Handbooks, 22

Branch, D., Parrent, J., Troxel, M. A., et al. 2007, in AIP Conf. Proc., Vol. 924 The Multicolored Landscape of Compact Objects and Their Explosive Origins, ed. L. A. Antonelli et al. (New York: AIP), 342

Bufano, F., Pignata, G., Bersten, M., et al. 2014, MNRAS, 439, 1807

Cappellaro, E., Mazzali, P. A., Benetti, S., et al. 1997, A\&A, 328, 203

Cardelli, J. A., Clayton, G. C., \& Mathis, J. S. 1989, ApJ, 345, 245

Chevalier, R. A., \& Fransson, C. 2006, ApJ, 651, 381

Chevalier, R. A., \& Soderberg, A. M. 2010, ApJL, 711, L40

Ciabattari, F., Mazzoni, E., Donati, S., et al. 2013, CBET, 3557, 1

Crockett, R. M., Eldridge, J. J., Smartt, S. J., et al. 2008, MNRAS, 391, L5

Ensman, L., \& Woosley, S. E. 1987, BAAS, 19, 757

Ergon, M., Jerkstrand, A., Sollerman, J., et al. 2014, arXiv:1408.0731

Ergon, M., Sollerman, J., Fraser, M., et al. 2014, A\&A, 562, A17

Evans, R. O., White, B., \& Bembrick, C. 2001, IAUC, 7772, 1

Filippenko, A. V. 1988, AJ, 96, 1941

Filippenko, A. V. 1997, ARA\&A, 35, 309

Filippenko, A. V., Matheson, T., \& Ho, L. C. 1993, ApJL, 415, L103

Fox, O. D., Bostroem, K. A., Van Dyk, S. D., et al. 2014, ApJ, 790, 17

Fransson, C., Lundqvist, P., \& Chevalier, R. A. 1996, ApJ, 461, 993

Freedman, W. L., Hughes, S. M., Madore, B. F., et al. 1994, ApJ, 427, 628

Freedman, W. L., Madore, B. F., Gibson, B. K., et al. 2001, ApJ, 553, 47

Gal-Yam, A., Arcavi, I., Ofek, E. O., et al. 2014, Natur, 509, 471

Georgy, C. 2012, A\&A, 538, L8

Hachinger, S., Mazzali, P. A., Taubenberger, S., et al. 2012, MNRAS, 422, 70

Hamuy, M., Deng, J., Mazzali, P. A., et al. 2009, ApJ, 703, 1612

Horesh, A., Stockdale, C., Fox, D. B., et al. 2013, MNRAS, 436, 1258

Jeffery, D. J., Kirshner, R. P., Challis, P. M., et al. 1994, ApJL, 421, L27

Keyes, C. D. 1995, Faint Object Spectrograph Instrument Handbook, HST Instrument Handbook, 47

Kirshner, R. P., Jeffery, D. J., Leibundgut, B., et al. 1993, ApJ, 415, 589

Law, N. M., Kulkarni, S. R., Dekany, R. G., et al. 2009, PASP, 121, 1395

Lucy, L. B. 1991, ApJ, 383, 308

Lucy, L. B. 1999, A\&A, 345, 211

Marion, G. H., Vinko, J., Kirshner, R. P., et al. 2014, ApJ, 781, 69

Maund, J. R., Fraser, M., Ergon, M., et al. 2011, ApJL, 739, L37

Maund, J. R., Smartt, S. J., Kudritzki, R. P., Podsiadlowski, P., \& Gilmore, G. F. 2004, Natur, 427, 129

Maurer, I., Mazzali, P. A., Taubenberger, S., \& Hachinger, S. 2010, MNRAS, 409, 1441

Mazzali, P. A. 2000, A\&A, 363, 705

Mazzali, P. A., Deng, J., Hamuy, M., \& Nomoto, K. 2009, ApJ, 703, 1624

Mazzali, P. A., Lucy, L. B., Danziger, I. J., et al. 1993, A\&A, 269, 423

Mazzali, P. A., Sullivan, M., Hachinger, S., et al. 2014, MNRAS, 439, 1959

Morales-Garoffolo, A., Elias-Rosa, N., Benetti, S., et al. 2014, arXiv: 1409.2784

Moriya, T. J., \& Tominaga, N. 2012, ApJ, 747, 118 
Nakano, S. 2008, CBET, 1286, 1

Nakar, E., \& Piro, A. L. 2014, ApJ, 788, 193

Ofek, E. O., Lin, L., Kouveliotou, C., et al. 2013, ApJ, 768, 47

Panagia, N. 2007, in AIP Conf. Proc. Vol. 937, Supernova 1987 A: 20 Years After: Supernovae \& Gamma-Ray Bursters, ed. S. Immler, \& K. Weiler (New York: AIP), 236

Parrent, J., Branch, D., \& Jeffery, D. 2010, ascl soft, 10055

Pastorello, A., Kasliwal, M. M., Crockett, R. M., et al. 2008, MNRAS, 389, 955

Perets, H. B., Gal-Yam, A., Mazzali, P. A., et al. 2010, Natur, 465, 322

Rau, A., Kulkarni, S. R., Law, N. M., et al. 2009, PASP, 121, 1334

Richmond, M. W., Treffers, R. R., Filippenko, A. V., \& Paik, Y. 1996, AJ, 112,732

Roming, P. W. A., Pritchard, T. A., Brown, P. J., et al. 2009, ApJL, 704, L118

Ryder, S. D., Murrowood, C. E., \& Stathakis, R. A. 2006, MNRAS, 369 L32

Ryder, S. D., Sadler, E. M., Subrahmanyan, R., et al. 2004, MNRAS, 349, 1093

Sauer, D. N., Mazzali, P. A., Blondin, S., et al. 2008, MNRAS, 391, 1605

Silverman, J. M., Mazzali, P., Chornock, R., et al. 2009, PASP, 121, 689

Silverman, J. M., Nugent, P. E., Gal-Yam, A., et al. 2013, ApJS, 207, 3

Smartt, S. J. 2009, ARA\&A, 47, 63

Smith, N., \& Conti, P. S. 2008, ApJ, 679, 1467
Smith, N., Li, W., Filippenko, A. V., \& Chornock, R. 2011, MNRAS, 412, 1522

Soderberg, A. M., Chevalier, R. A., Kulkarni, S. R., \& Frail, D. A. 2006, ApJ, 651,1005

Soria, R., Kuncic, Z., Broderick, J. W., \& Ryder, S. D. 2006, MNRAS, 370,1666

Stancliffe, R. J., \& Eldridge, J. J. 2009, MNRAS, 396, 1699

Stehle, M., Mazzali, P. A., Benetti, S., \& Hillebrandt, W. 2005, MNRAS, 360, 1231

Sugimoto, D., \& Nomoto, K. 1980, SSRv, 25, 155

Tsvetkov, D. Y., Volkov, I. M., Baklanov, P., Blinnikov, S., \& Tuchin, O. 2009, PZ, 29, 2

Van Dyk, S. D., Garnavich, P. M., Filippenko, A. V., et al. 2002, PASP, 114,1322

Van Dyk, S. D., Li, W., Cenko, S. B., et al. 2011, ApJL, 741, L28

Van Dyk, S. D., Weiler, K. W., Sramek, R. A., Rupen, M. P., \& Panagia, N. 1994, ApJL, 432, L115

Van Dyk, S. D., Zheng, W., Clubb, K. I., et al. 2013, ApJL, 772, L32

Van Dyk, S. D., Zheng, W., Fox, O. D., et al. 2014, AJ, 147, 37

Woosley, S. E., Eastman, R. G., Weaver, T. A., \& Pinto, P. A. 1994, ApJ, 429, 300

Woosley, S., \& Janka, T. 2005, NatPh, 1, 147

Yaron, O., \& Gal-Yam, A. 2012, PASP, 124, 668 